\title{
S-Doped $\mathrm{ZnSnO}_{3}$ Nanoparticles with Narrow Band Gaps for Photocatalytic Wastewater Treatment
}

Runjiang $\mathrm{Guo}^{\dagger}$, Ran Tian ${ }^{\dagger}$, Dongliang Shi ${ }^{\ddagger}$, Hua Li*,†, Hezhou Liu ${ }^{\dagger}$

†State Key Laboratory of Metal Matrix Composites, School of Materials Science and Engineering, Shanghai Jiaotong University, Shanghai 200240, China

$₫$ Xinhua Hospital Affiliated to Medicine School of Shanghai Jiaotong University, Shanghai 200092, China

*Email address:lih@sjtu.edu.cn

Tel.: +862134202549 


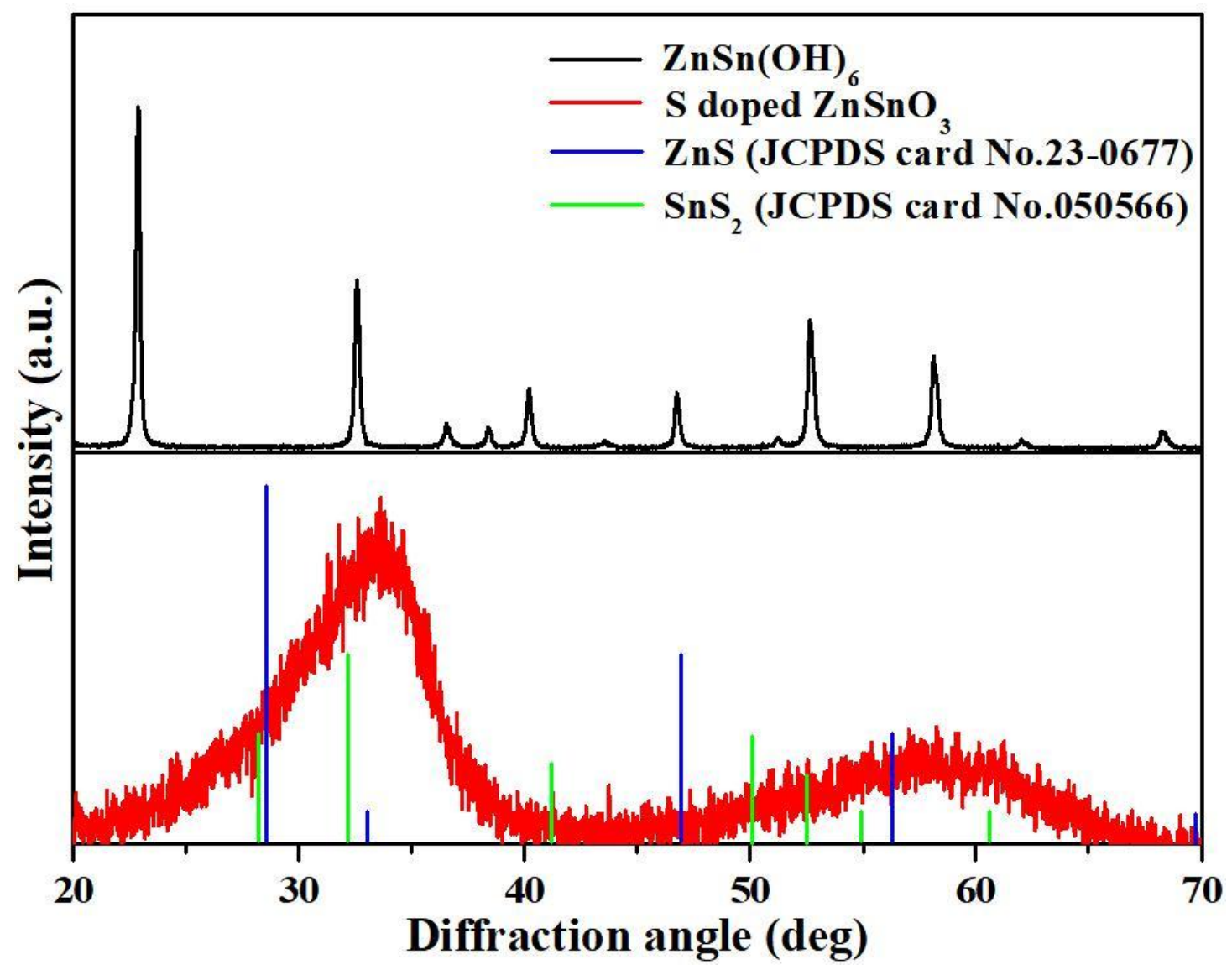

Figure $\mathrm{S1}$ The $\mathrm{XRD}$ pattern of $\mathrm{ZnSn}(\mathrm{OH})_{6}$ and $\mathrm{S}$ doped $\mathrm{ZnSnO}_{3}$ as well as the standard JCPDS card of $\mathrm{ZnS}$ and $\mathrm{SnS}_{2}$.

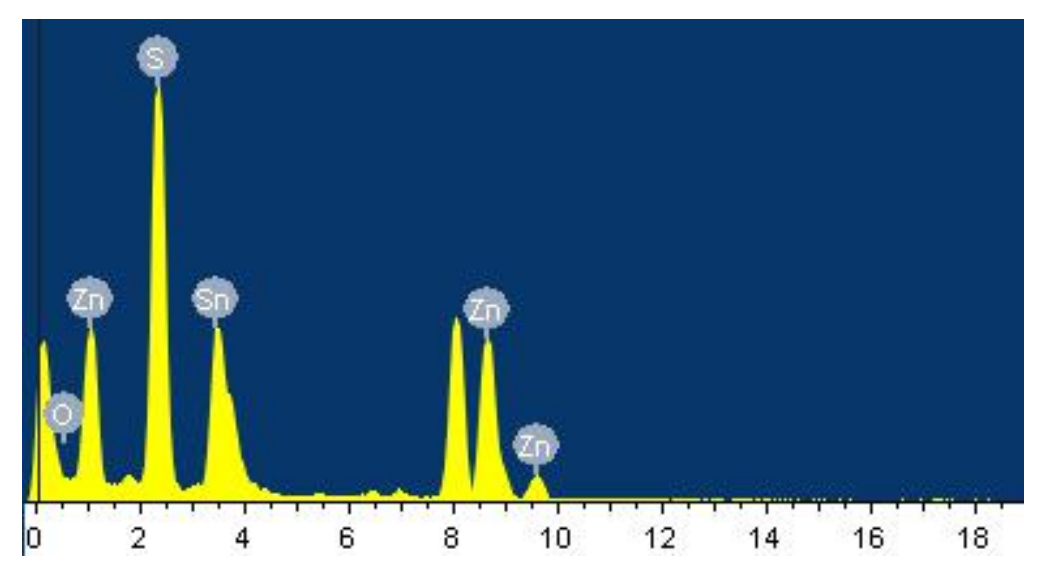

Figure $\mathrm{S} 2$ The EDS pattern of $\mathrm{S}$ doped $\mathrm{ZnSnO}_{3}$. 

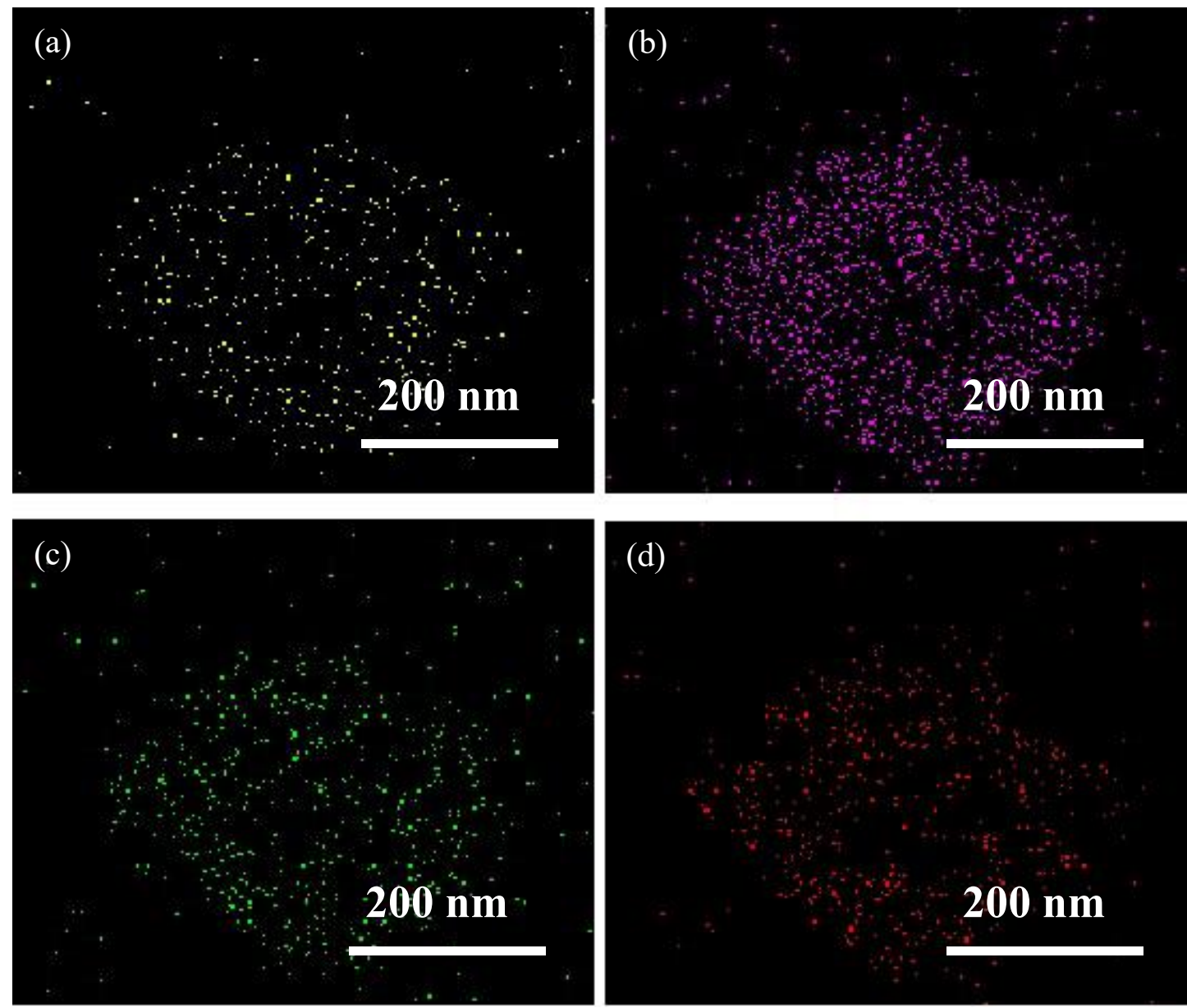

Figure $\mathrm{S3}$ The corresponding elements mapping of $\mathrm{S}$ doped $\mathrm{ZnSnO}_{3}$ based on Figure 3 (d) with the elements of (a) O, (b) S, (c) Zn and (d) Sn.
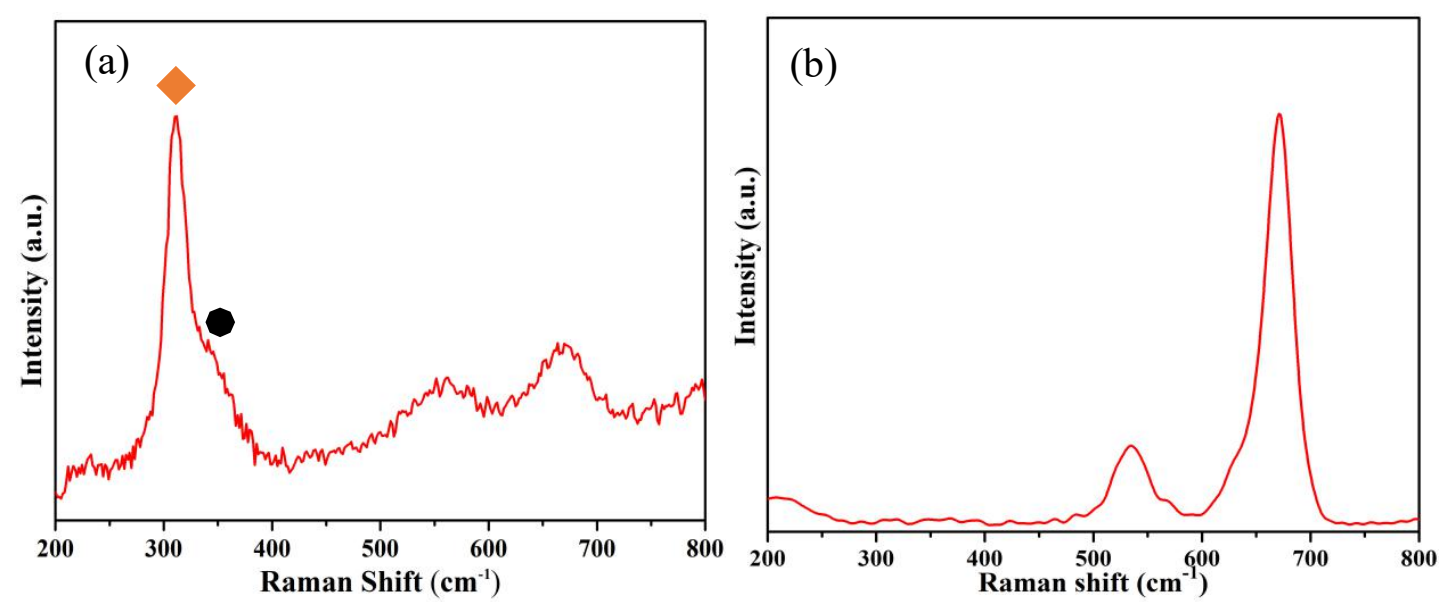

Figure S4 The Raman spectra of (a) $\mathrm{S}$ doped $\mathrm{ZnSnO}_{3}$ and (b) pure $\mathrm{ZnSnO}_{3}$. 

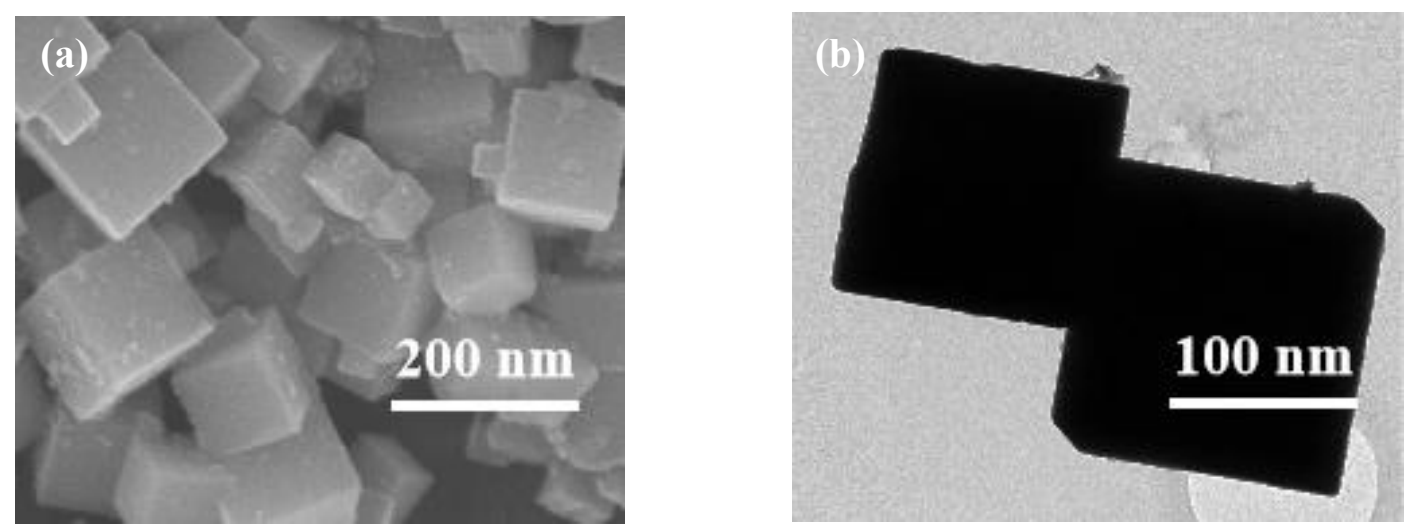

Figure S5 Typical (a) SEM, (b) TEM images of as synthesized $\mathrm{ZnSnO}_{3}$ nanocubes.
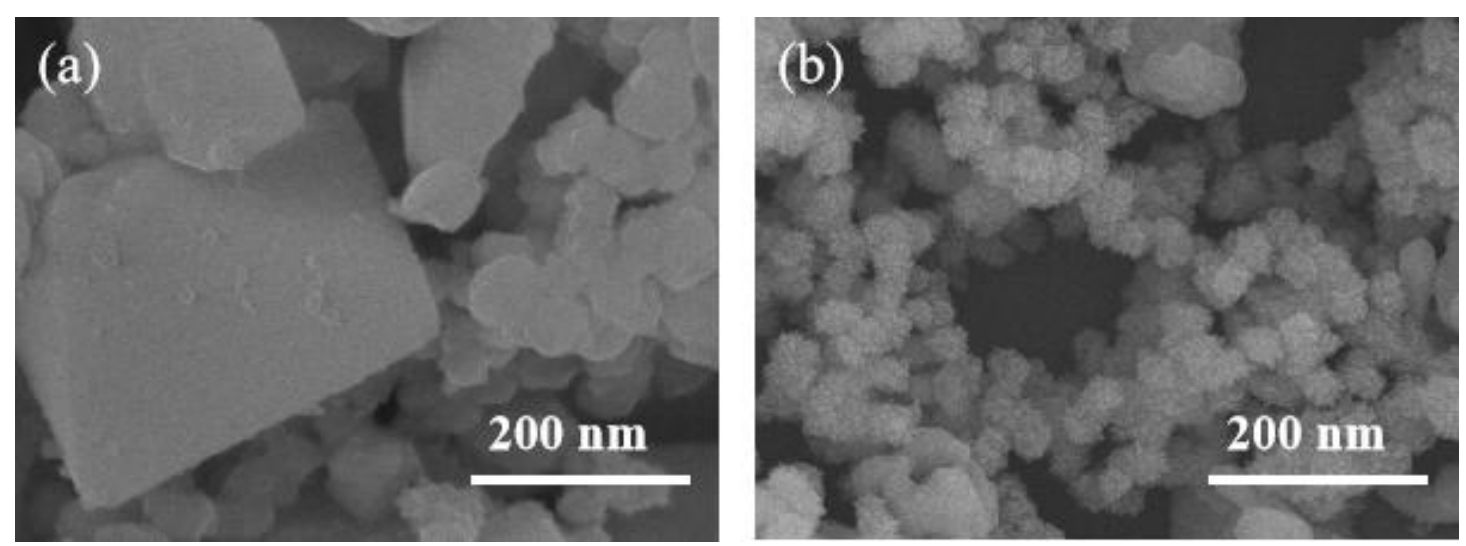

Figure S6 The SEM images of $\mathrm{ZnSn}(\mathrm{OH})_{6}$ with the volume ratio of ethanol and water (a) 20/20 and (b) 10/30. 

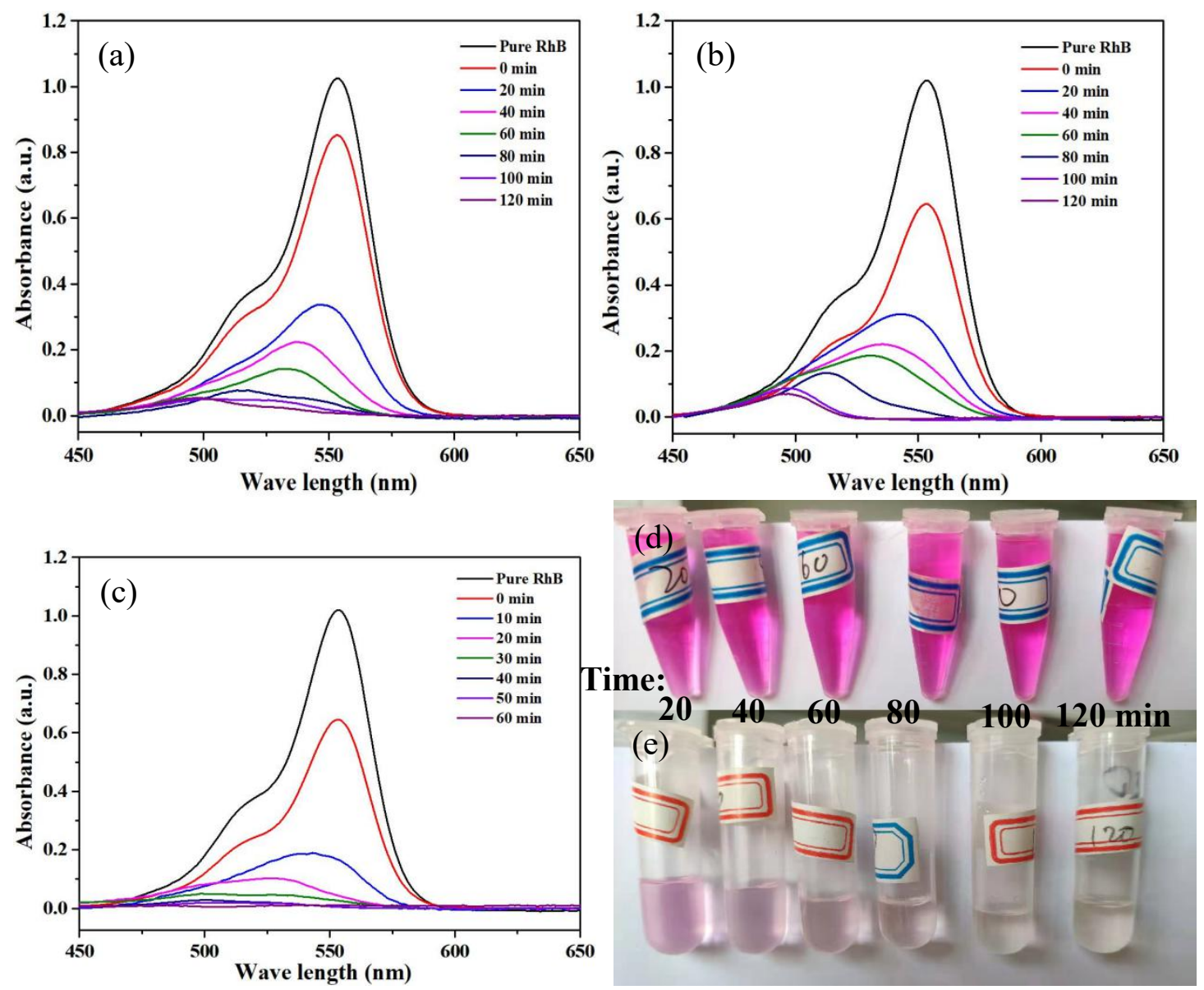

Figure $\mathrm{S} 7$ The full spectrum light absorption spectra of (a) $\mathrm{ZnSnO}_{3}$ and (b) $\mathrm{S}$ doped $\mathrm{ZnSnO}_{3}$; the visible light absorption spectra of (c) $\mathrm{S}$ doped $\mathrm{ZnSnO}_{3}$; the color photos taken every 20 min during the visible-light photo-degradation processes of (d) pure $\mathrm{ZnSnO}_{3}$ and (e) $\mathrm{S}_{\text {-doped }} \mathrm{ZnSnO}_{3}$ respectively. 

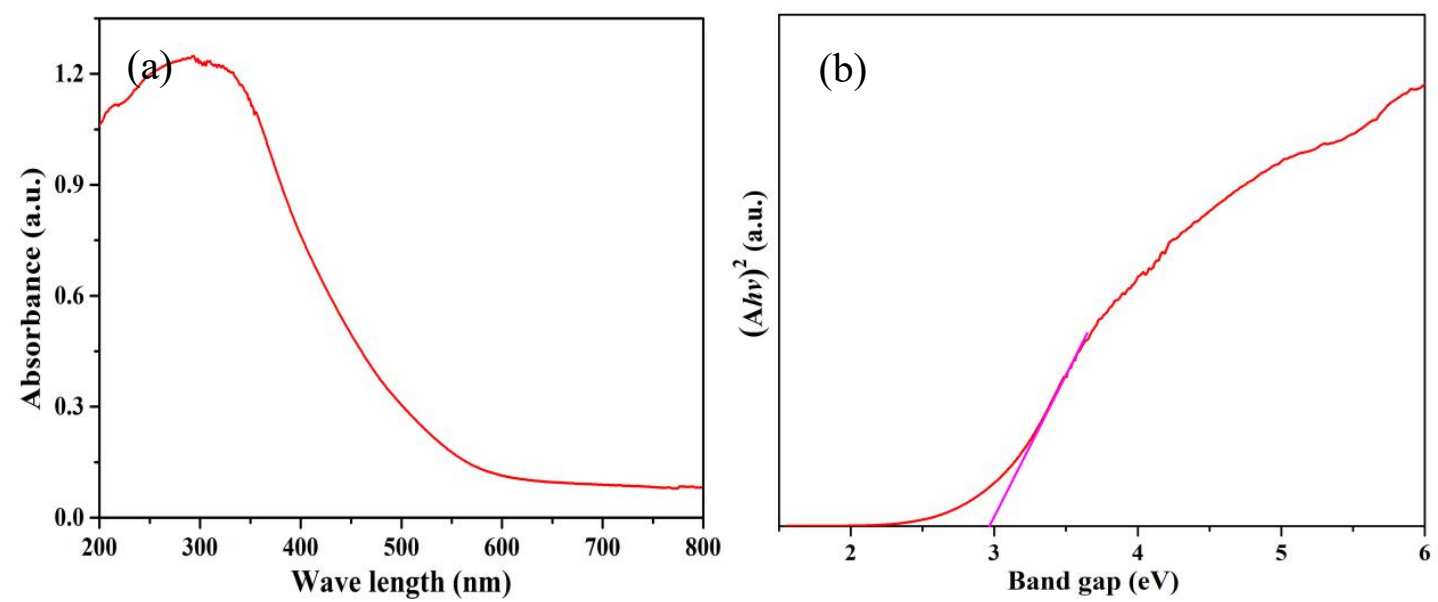

Figure S8 (a) The UV-vis diffuse reflectance spectra of $\mathrm{S}$ doped solid $\mathrm{ZnSnO}_{3}$; (b) the corresponding Kubelka-Munk transformed reflectance spectra to calculate the band gap.
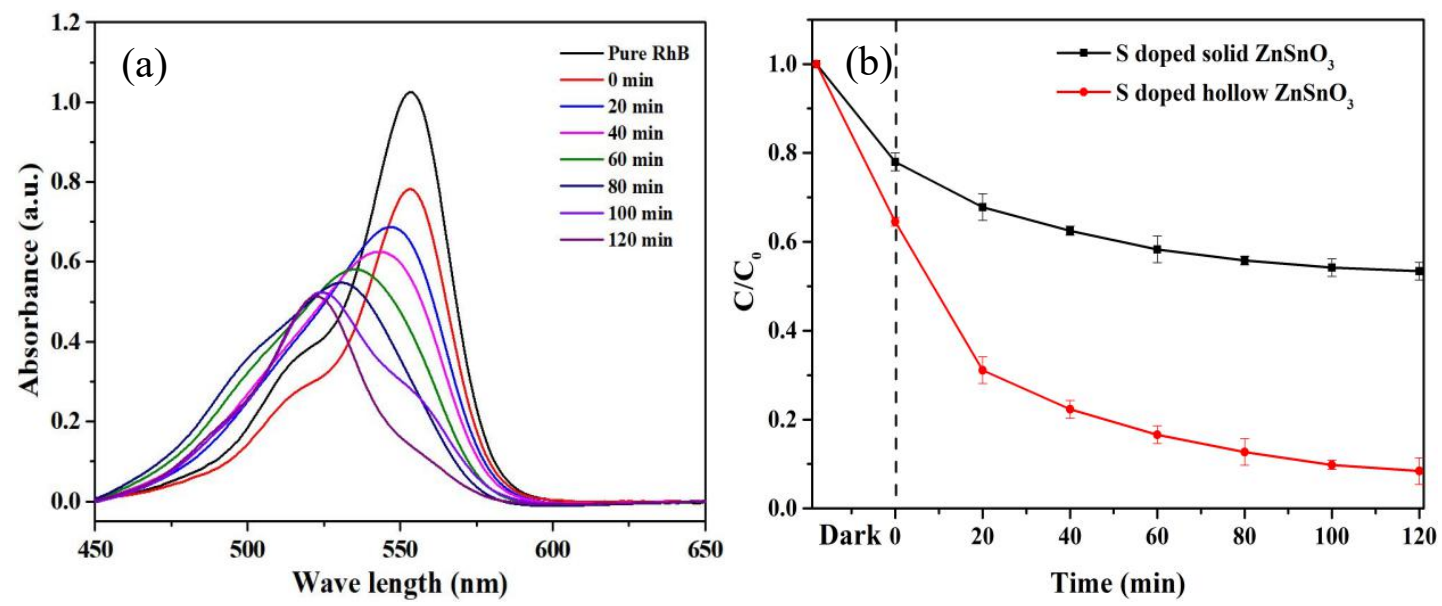

Figure S9 (a) The visible light photodegradation efficiency of $S$ doped solid $\mathrm{ZnSnO}_{3}$ shown by UV-vis absorption spectra. (b) The photodegradation efficiency of $\mathrm{S}$ doped solid and hollow $\mathrm{ZnSnO}_{3}$ under visible light respectively. 

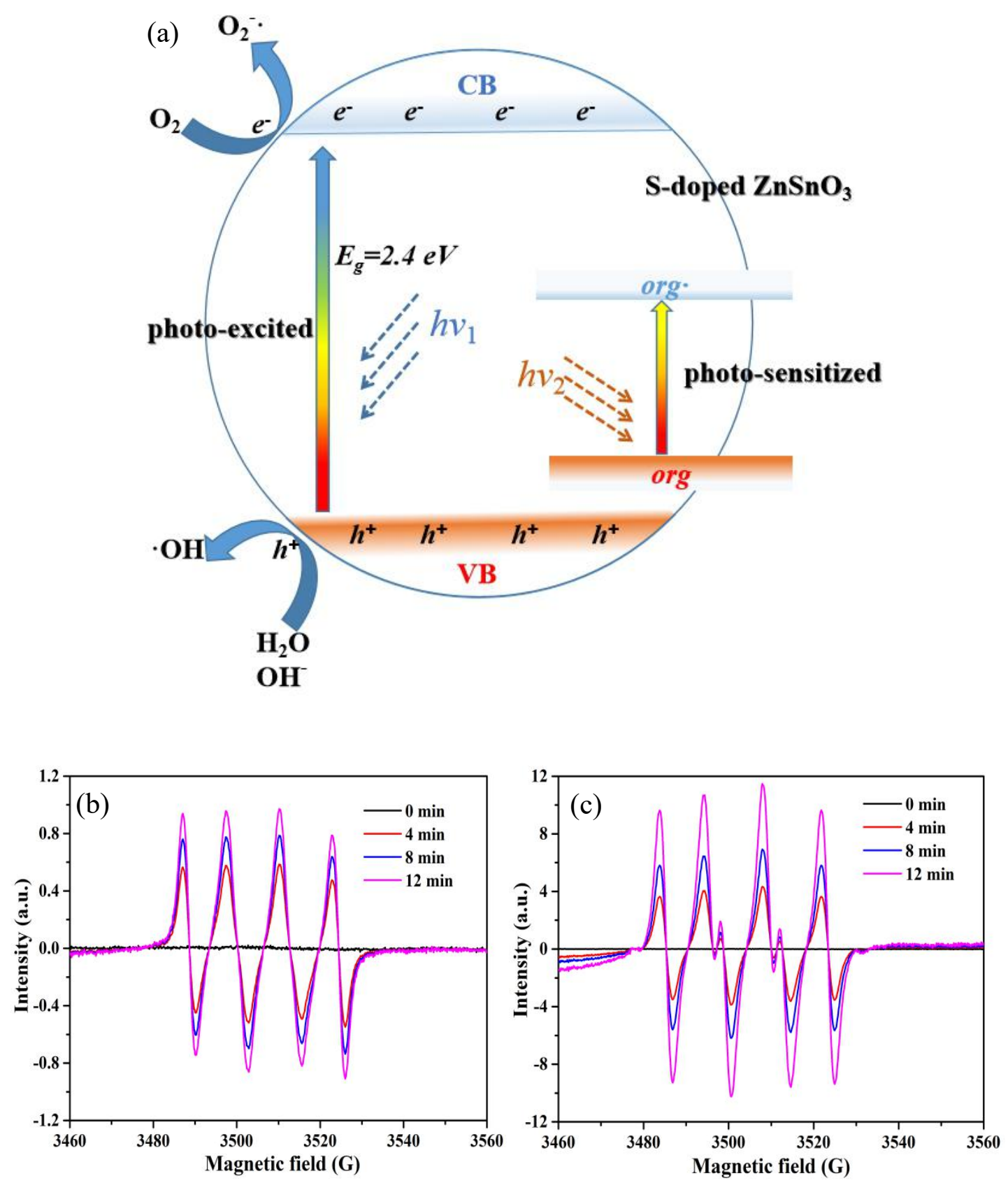

Figure S10 (a) The working mechanism of RhB photodegradation via S doped $\mathrm{ZnSnO}_{3}$; the ESR spectra of $\mathrm{DMPO}-\bullet \mathrm{O}^{2-}$ for (b) $\mathrm{S}$-doped $\mathrm{ZnSnO}_{3}$ and (c) $\mathrm{ZnSnO}_{3}$. 
Table S1 The performances of photocatalysts based on different zinc-tin-oxide composite materials.

\begin{tabular}{|c|c|c|c|c|}
\hline Photocatalyst & Morphology & $\begin{array}{l}\text { Efficiency } \\
\text { (UV light)* }\end{array}$ & $\begin{array}{l}\text { Efficiency } \\
\text { (visible light)* }\end{array}$ & Ref. \\
\hline $\mathrm{C}$ doped $\mathrm{Zn}_{2} \mathrm{SnO}_{4}$ & Nanocubes & 60 & $* *$ & S1 \\
\hline Defective $\mathrm{Zn}_{2} \mathrm{SnO}_{4}$ & Nanoparticles & $* *$ & Over 120 & S2 \\
\hline ZnSn(OH)6@BiOI & $\begin{array}{l}\text { Heterojunction } \\
\text { structures }\end{array}$ & 40 & Over 120 & S3 \\
\hline $\mathrm{Zn}_{2} \mathrm{SnO}_{4} @ \mathrm{SnO}_{2}$ & $\begin{array}{l}\text { Heterojunction } \\
\text { structures }\end{array}$ & 45 & $* * *$ & S4 \\
\hline ZnSn(OH)6@BiOCl & $\begin{array}{l}\text { Heterojunction } \\
\text { structure }\end{array}$ & $* *$ & 120 & S5 \\
\hline $\mathrm{Zn}_{2} \mathrm{SnO}_{4}$ & Octahedra & 60 & $* * *$ & S6 \\
\hline $\mathrm{ZnSn}(\mathrm{OH})_{6}$ & Hollow cubes & 80 & $* * *$ & S7 \\
\hline $\mathrm{ZnSnO}_{3} @ \mathbf{r G O}$ & Hollow spheres & 15 & Over 180 & S8 \\
\hline $\mathrm{S}$ doped $\mathrm{Zn}_{2} \mathrm{SnO}_{4}$ & Nanoparticles & $* *$ & $\leq 64 \%$ & S9 \\
\hline $\mathrm{ZnSnO}_{3}$ & Nanocubes & 75 & $* * *$ & S10 \\
\hline $\mathrm{S}$ doped $\mathrm{ZnSnO}_{3}$ & $\begin{array}{l}\text { Hierarchical } \\
\text { structures }\end{array}$ & 20 & 80 & $\begin{array}{l}\text { This } \\
\text { work }\end{array}$ \\
\hline
\end{tabular}

* The photocatalysis efficiency is defined as the time (minutes) it takes when about $90 \%$ dye has been degraded.

** The photocatalysis efficiency under visible light or UV light is not mentioned in related reference.

$* * *$ The materials have no photocatalysis efficiency under visible light due to their wide band gap. 


\section{References}

[S1] Li, H.; Hong, W.; Cui, Y.; Jia, Q.; Fan, S. High Photocatalytic Activity of

C-ZnSn(OH) 6 Catalysts Prepared by Hydrothermal Method. J. Mol. Catal. A-Chem. 2013, 378, 164-173.

[S2] Ben-Ali, M.; Barka-Bouaifel, F.; Elhouichet, H.; Sieber, B.; Addad, A.; Boussekey, L.; Férid, M.; Boukherroub, R. Hydrothermal Synthesis, Phase Structure, Optical and Photocatalytic Properties of $\mathrm{Zn}_{2} \mathrm{SnO}_{4}$ Nanoparticles. J. Colloid Interf. Sci. $2015,457,360-369$.

[S3] Li, H.; Cui, Y.; Hong, W.; Xu, B. Enhanced Photocatalytic Activities of $\mathrm{BiOI} / \mathrm{ZnSn}(\mathrm{OH})_{6}$ Composites Towards the Degradation of Phenol and Photocatalytic $\mathrm{H}_{2}$ Production. Chem. Eng. J. 2013, 228, 1110-1120.

[S4] Liu, C.; Röder, R.; Zhang, L.; Ren, Z.; Chen, H.; Zhang, Z.; Ronning, C.; Gao, P. Highly Efficient Visible-Light Driven Photocatalysts: a Case of Zinc Stannate Based Nanocrystal Assemblies. J. Mater. Chem. A 2014, 2, 4157-4167.

[S5] Wang, H.; Yuan, X.; Wu, Y.; Zeng, G.; Tu, W.; Sheng, C.; Deng, Y.; Chen, F.; Chew, J. Plasmonic Bi nanoparticles and BiOCl Sheets as Cocatalyst Deposited on Perovskite-Type $\mathrm{ZnSn}(\mathrm{OH})_{6}$ Microparticle with Facet-Oriented Polyhedron for Improved Visible-Light-Driven Photocatalysis. Appl. Catal. B-Environ. 2017, 209, 543-553.

[S6] Zhao, Q.; Ju, D.; Song, X.; Deng, X.; Ding, M.; Xu, X.; Zeng, H. Polyhedral $\mathrm{Zn}_{2} \mathrm{SnO}_{4}$ : Synthesis, Enhanced Gas Sensing and Photocatalytic Performance. Sensor Actuat. B-Chem. 2016, 229, 627-634.

[S7] Fu, X.; Huang, D.; Qin, Y.; Li, L.; Jiang, X.; Chen, S. Effects of Preparation Method on the Microstructure and Photocatalytic Performance of $\mathrm{ZnSn}(\mathrm{OH})_{6}$, Appl. Catal. B-Environ. 2014, 148-149, 532-542.

[S8] Dong, S.; Sun, J.; Li, Y.; Yu, C.; Li, Y.; Sun, J. $\mathrm{ZnSnO}_{3}$ Hollow Nanospheres/Reduced Graphene Oxide Nanocomposites as High-Performance Photocatalysts for Degradation of Metronidazole. Appl. Catal. B-Environ. 2014, 144 
386-393.

[S9] Lin, Y.; Lin, S.; Luo, M.; Liu, J. Enhanced Visible Light Photocatalytic Activity of $\mathrm{Zn}_{2} \mathrm{SnO}_{4}$ via Sulfur Anion-Doping. Mater. Lett. 2009, 63, 1169-1171.

[S10] Huang, J.; Xu, X.; Gu, C.; Wang, W.; Geng, B.; Sun, Y.; Liu, J. Size-Controlled Synthesis of Porous $\mathrm{ZnSnO}_{3}$ Cubes and their Gas-sensing and Photocatalysis Properties. Sensor Actuat. B-Chem. 2012, 171-172, 572-579. 